\title{
Traumatismo craneoencefálico en niños. Hospital General de Medellín y Clínica Somer de Rionegro, 2010-2017
}

\author{
Diana Paola Jiménez-Aguilar', Luisa María Montoya-Jaramillo', Dione Benjumea-Bedoya², \\ John Fredy Castro-Álvarez ${ }^{3}$
}

\section{RESUMEN}

Objetivo: describir las características sociodemográficas, aspectos clínicos y complicaciones de los niños de 0 a 10 años de edad que sufrieron traumatismo craneoencefálico, atendidos en el Hospital General de Medellín y en la Clínica Somer de Rioneogro entre los años 2010 y 2017.

Métodos: estudio descriptivo retrospectivo, toma como fuente de información las historias clínicas de los niños con diagnósticos relacionados con el traumatismo craneoencefálico entre 2010-2017. Se calcularon las proporciones de las variables sociodemográficas, circunstanciales, espacio-temporales y clínicas.

Resultados: se encontraron 224 pacientes con traumatismo craneoencefálico, el 64,7 \% de los casos fueron de sexo masculino, la edad promedio fue de 4,5 años. El trauma ocurrió con mayor frecuencia en el domicilio del paciente entre los días de la semana en las horas de la tarde. La causa principal del trauma fue caída (75\%), seguido por accidentes de tránsito (13,3 \%). La gravedad del traumatismo se midió con la escala de coma de Glasgow, el 78 \% fue leve, hubo un caso fatal y 7 (3\%) tuvieron complicaciones motoras durante la hospitalización.

Conclusión: los hallazgos de este estudio coinciden con las principales características del traumatismo craneoencefálico de la población pediátrica en el mundo, amplía la información regional y local para el desarrollo de estrategias de prevención, diagnóstico y seguimiento a largo plazo de los pacientes.

\footnotetext{
MD, Residente de tercer año de Pediatría, Corporación Universitaria Remington, Medellín, Colombia.

MD. Mg. Epidemiología PhD. Grupo de Investigaciones Biomédicas, Facultad de Ciencias de la Salud, Corporación Universitaria Remington, Medellín, Colombia.

MB. MSc. PhD. Grupo de Investigación en Salud del Adulto Mayor, Facultad de Ciencias de la Salud, Corporación Universitaria Remington, Medellín, Colombia.
}

Correspondencia: Diana Paola Jiménez-Aguilar; floradejade@hotmail.com

Recibido: abril 23 de 2019

Aceptado: julio 19 de 2019

Cómo citar: Jiménez-Aguilar DP, Montoya-Jaramillo LM, Benjumea-Bedoya D, Castro-Álvarez JF. Traumatismo craneoencefálico en niños. Hospital General de Medellín y Clínica Somer de Rionegro, 2010-2017. latreia. 2020 Ene-Mar;33(1):28-38. 2019. DOl 10.17533/udea.iatreia.36. 


\section{PALABRAS CLAVE}

Cráneo; Encéfalo; Niño; Traumatismo Craneoencefálico

\section{SUMMARY}

Pediatric Traumatic Brain Injury. Hospital General of Medellin and Clínica Somer of Rionegro, 20102017

Objective: To describe the demography, clinical presentation and complications associated with traumatic brain injury (TBI) in a cohort of children, ages 0-10, treated at Hospital General de Medellín and Clínica Somer between 2010-2017.

Methods: Cross sectional retrospective study chart review of all children with a diagnosis of TBI between 2010 and 2017. There were systematic review and recording of demographic information, mechanism of injury, clinical presentation, and associated complications.

Results: A total of 224 patients with TBI were identified. Among them, $64.7 \%$ were males and mean age was 4.5 years. Injuries most frequently occurred at the patient's home, during the afternoon hours and between Monday and Friday. The most common mechanisms of injury were falls ( $75 \%)$, followed by motor vehicle accidents (13.3\%). Severity of injury, measured by Glasgow coma scale, found that $78 \%$ of cases were mild. Seven $(3 \%)$ of cases had neuromuscular complications during hospitalization and one TBI related death was identified.

Conclusion: Findings of this study are consistent with the known characteristics of pediatric TBI worldwide. This information may be used to improve prevention, diagnosis, and long term follow-up.

\section{KEY WORDS}

Brain; Brain Injuries, Traumatic; Child; SkuII

\section{INTRODUCCIÓN}

EI traumatismo craneoencefálico (TCE) es la alteración en la función neurológica causada por una fuerza externa que ocasiona un daño físico en el cerebro ${ }^{(1,2)}$. La etiología varía según la edad y se relaciona con el grado de desarrollo psicomotor del niño. Entre los principales mecanismos de lesiones están las caídas, los accidentes de tránsito, los traumas directos y el maltrato físico ${ }^{(3-8)}$, lo anterior sumado a factores de riesgo del entorno de los niños como el abandono, el nivel de escolaridad y la condición económica de los cuidadores ${ }^{\left({ }^{9}\right.}$. EI TCE puede clasificarse según el tipo de lesión en contusiones, fracturas craneanas y hemorragias intracraneanas o, según la gravedad de acuerdo con la escala de coma de Glasoow, adaptada a la edad pediátrica así: TCE leve con puntaje entre 13 y 15 , TCE moderado con puntaje entre 9 y 12 y TCE grave con puntaje menor o igual a $8^{(2,10)}$.

EI TCE es una causa importante de discapacidad y muerte ${ }^{(5,1)}$, la incidencia mundial varía según el país, en un rango que va desde $47-280$ por cada 100.000 niños ${ }^{(12)}$, donde el $80 \%$ de los TCE son leves con una mortalidad entre el 1-6\% ${ }^{(13,14)}$. El pronóstico depende de la gravedad del trauma inicial, las lesiones graves se asocian con el riesgo de desarrollar discapacidad permanente o riesgo de muerte. Los pacientes con traumas leves o moderados pueden tener secuelas motrices y cognitivas a largo plazo ${ }^{(13,15-17)}$. Esta condición demanda altos costos al sistema de salud, ya que cada año alrededor de 10 millones de niños en Estados Unidos requieren hospitalización como consecuencia del TCE ${ }^{(18,19)}$. Los niños de 0 a 4 años de edad presentan las tasas más altas de consultas a los servicios de urgencias relacionadas con TCE en dicho país ${ }^{(20)}$.

En Colombia no existe un registro nacional de la incidencia del TCE, si bien se han realizado estudios que permiten hacer una aproximación a las características epidemiológicas, estos resultados no se logran extrapolar a la población infantil en el país ${ }^{(21-25)}$. Con respecto a Medellín, en el año 2000 el TCE representó la segunda causa de consulta en los servicios de urgencias pediátricos con niños menores de 4 años y la principal causa de muerte en la población entre 1 y 14 años de edad ${ }^{(26)}$. En el año 2008 se describieron algunas características sociodemográficas y clínicas de niños entre 1 y 5 años que sufrieron TCE $y$ fueron atendidos en un instituto de salud de alta complejidad de la ciudad ${ }^{(24)}$, Io anterior hace 
necesario más estudios locales que procuren una caracterización más amplia del TCE en la población infantil. Es por esto que en este artículo se describen las características sociodemográficas, clínicas, espacio-temporales y circunstanciales; asociadas a la gravedad deI TCE en pacientes de 0 a 10 años de edad que fueron atendidos en dos instituciones de salud de alta complejidad.

\section{MATERIALES Y MÉTODOS}

Estudio descriptivo y retrospectivo que recoge información de fuentes secundarias. El periodo de estudio fue entre los años 2010-2017, se realizó un análisis de la historia clínica de los pacientes de 0 a 10 años de edad, con diagnóstico de TCE atendidos en el Hospital General de Medellín y en la Clínica Somer de Rionegro. Se buscaron diagnósticos relacionados con el TCE, se hizo uso de los códigos S000, S010, S020, S021, S027, S061-S065, S080, I608, I609 de Ia Clasificación Estadística Internacional de Enfermedades y Problemas Relacionados con Ia Salud CIE 10 (27). Se creó una base de datos en Microsoft Excel en la que se registraron las variables sociodemográficas, circunstanciales, espacio-temporales y clínicas.

Se aplicó la estadística descriptiva y se calcularon las proporciones de las variables mencionadas antes. La variable edad fue categorizada en los siguientes grupos: Lactantes de 0 a 2 años; Preescolares de 3 a 5 años y Escolares de 6 a 10 años ${ }^{(28)}$. El estrato socioeconómico en Colombia lo define la empresa proveedora de servicios públicos, este se categorizó en los siguientes grupos: Nivel bajo (estratos 1 y 2), Nivel medio (estratos 3 y 4) y Nivel alto (estratos 5 y 6). El análisis bivariado se hizo según la severidad del TCE, categorizado como Leve y Moderado/Grave, se recategorizó también la variable del estrato socioe-

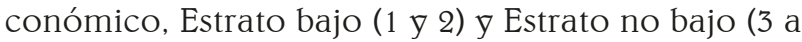
6), Ias demás variables incluidas fueron sexo, edad, sitio de atención y tener acompañante. Se utilizó la prueba chi-cuadrado de independencia para explorar diferencias entre las variables, con una confianza del $95 \%$.

Esta investigación fue aprobada por el Comité de Ética en Investigación de la Clínica Somer (acta 16 del 11 de diciembre de 2017) y por el Hospital General de
Medellín (acta 04-04042017 del 04 de abril de 2017). Se clasificó sin riesgo según la Resolución 8430 de 1993 del Ministerio de Salud de Colombia.

\section{RESULTADOS}

En el periodo de estudio comprendido entre 2010 y 2017 se identificaron en total 224 historias clínicas, de las cuales 95 casos (42,4\%) fueron atendidos en el Hospital General de Medellín y 129 casos (57,5 \%) en la Clínica Somer de Rionegro. En los municipios donde más se presentó el evento fue en Rionegro, con 68 registros que representan el $30,3 \%$ de los casos, seguido de Medellín con 55 (24,5\%) y Marinilla con 30 casos (13,3\%). El sexo masculino predominó con 145 casos $(64,7 \%)$, hubo una distribución similar en los tres grupos de edad, la media de la edad fue 4,5 años (DE 3,1$)$. Noventa pacientes $(40,1 \%)$ pertenecían al nivel socioeconómico bajo y 133 (59,3\%) al nivel socioeconómico medio; el sitio donde ocurrió el mayor número de traumas fue en el domicilio con 126 casos que representan el 56,3 \% (Tabla 1).

En el momento del trauma 198 pacientes $(88,3 \%)$ se encontraban acompañados, de ellos 144 (64,2\%) estaban bajo el cuidado de la madre (Tabla 2). Las causas de lesión que predominaron fueron las caídas con un total de 186 ( $83 \%$ ), seguido de accidentes de tránsito y traumas contusos (Tabla 2). El vehículo más implicado en los accidentes de tránsito fue la moto con 17 casos (56,6\%), seguido por el carro con 13 casos (43,3\%). De estos 17 niños se encontraban en calidad de peatones (56,6 \%), 9 niños en calidad de pasajeros (30\%), 3 niños en calidad de parrillero de moto $(10 \%)$ y un niño $(3,3 \%)$ en calidad de conductor de bicicleta.

Los principales tipos de diagnóstico según la lesión fueron traumatismos superficiales del cuero cabelludo en 102 pacientes (45,5\%) y hemorragias epidurales 34 pacientes $(15,1 \%)$ (Tabla 2$)$. Se clasificaron como TCE leve 175 casos $(78,1 \%)$, moderados 37 casos $(6,5 \%)$ y graves 12 casos $(5,3 \%)$. Del total de Ios casos estudiados uno falleció $(0,44 \%)$. De los 224 pacientes, $113(50,4 \%)$ requirieron observación en urgencias y $57(25,4 \%)$ ingresaron al servicio de hospitalización. El promedio de días totales de estancia hospitalaria fue 3,87 (DE 6,8) y en unidad de cuidados 
Tabla 1. Características sociodemográficas y espacio-temporales de los niños con traumatismo craneoencefálico.

Hospital General de Medellín y Clínica Somer de Rionegro, 2010-2017

\begin{tabular}{|c|c|c|c|}
\hline \multirow{2}{*}{ Característica } & & \multicolumn{2}{|c|}{ Registros evaluados } \\
\hline & & $n=224$ & $\%$ \\
\hline \multirow{3}{*}{ Edad } & $0-2$ años & 69 & 30,8 \\
\hline & 3-5 años & 65 & 29,0 \\
\hline & 6-10 años & 90 & 40,1 \\
\hline \multirow{2}{*}{ Sexo } & Femenino & 79 & 35,2 \\
\hline & Masculino & 145 & 64,7 \\
\hline \multirow{3}{*}{ Nivel socioeconómico } & Bajo & 90 & 40,1 \\
\hline & Medio & 133 & 59,3 \\
\hline & Alto & 1 & 0,4 \\
\hline \multirow{2}{*}{ Régimen de afiliación a salud } & Subsidiado & 71 & 31,6 \\
\hline & Contributivo & 153 & 68,3 \\
\hline \multirow{2}{*}{ Institución de salud } & Hospital General de Medellín & 95 & 42,4 \\
\hline & Clínica Somer de Rionegro & 129 & 57,5 \\
\hline \multirow{5}{*}{ Escolaridad } & No estudia & 80 & 35,7 \\
\hline & Prejardín & 17 & 7,5 \\
\hline & Jardín & 22 & 9,8 \\
\hline & Transición & 19 & 8,4 \\
\hline & Básica primaria & 86 & 38,3 \\
\hline \multirow{5}{*}{ Sitio de ocurrencia } & Domicilio & 126 & 56,2 \\
\hline & Vía pública & 55 & 24,5 \\
\hline & Parque infantil & 26 & 11,6 \\
\hline & Escuela & 7 & 3,1 \\
\hline & Otro & 10 & 45,4 \\
\hline \multirow{4}{*}{ Municipio de ocurrencia } & Rionegro & 68 & 30,3 \\
\hline & Medellín & 55 & 24,5 \\
\hline & Marinilla & 30 & 13,3 \\
\hline & Otro & 71 & 31,9 \\
\hline \multirow{2}{*}{$\begin{array}{l}\text { Periodo de la semana en que } \\
\text { ocurrió el evento }\end{array}$} & En semana & 158 & 70,5 \\
\hline & Fin de semana & 66 & 29,4 \\
\hline \multirow{3}{*}{ Jornada del día en que ocurrió } & Mañana & 56 & 25,0 \\
\hline & Tarde & 131 & 58,4 \\
\hline & Noche & 37 & 16,5 \\
\hline
\end{tabular}

Fuente: creación propia

intensivos o especiales fue 1,58 días (DE 4,2). Durante el tiempo de hospitalización se identificaron complicaciones motoras en 5 pacientes $(2,2 \%)$, motoras $y$ sensitivas en 4 pacientes (1,7\%), no tuvieron secuelas detectables durante la hospitalización 215 pacientes (95,9\%) (Tabla 2). 
Tabla 2. Características circunstanciales y clínicas de los niños con traumatismo craneoencefálico. Hospital General de Medellín y Clínica Somer de Rionegro, 2010-2017

\begin{tabular}{|c|c|c|c|}
\hline \multirow{2}{*}{\multicolumn{2}{|c|}{ Característica }} & \multicolumn{2}{|c|}{ Registros evaluados } \\
\hline & & $n=224$ & $\%$ \\
\hline \multirow{5}{*}{ Mecanismo de la lesión } & Caída & 186 & 83,0 \\
\hline & Accidente de tránsito & 30 & 13,4 \\
\hline & Maltrato físico & 3 & 1,3 \\
\hline & Trauma perinatal & 3 & 1,3 \\
\hline & Desconocido & 2 & 0,9 \\
\hline \multirow{9}{*}{ Acompañante en el momento del accidente } & Madre & 144 & 64,2 \\
\hline & Padre & 32 & 14,2 \\
\hline & Abuela/o & 13 & 5,8 \\
\hline & Tío/a & 6 & 2,6 \\
\hline & Hermano/a & 1 & 0,4 \\
\hline & Niñera & 1 & 0,4 \\
\hline & Profesor & 1 & 0,4 \\
\hline & Sin acompañante & 23 & 10,2 \\
\hline & Sin información & 3 & 1,3 \\
\hline \multirow{10}{*}{ Tipo de lesión } & Traumatismo superficial del cuero cabelludo & 102 & 45,5 \\
\hline & Hemorragia epidural & 34 & 15,1 \\
\hline & Fractura de la bóveda del cráneo & 28 & 12,5 \\
\hline & Hemorragia subdural traumática & 19 & 7,8 \\
\hline & Traumatismo cerebral difuso & 18 & 8,0 \\
\hline & Fractura hueso de cráneo & 8 & 3,5 \\
\hline & Fractura de la base del cráneo & 6 & 2,6 \\
\hline & Edema cerebral traumático & 4 & 1,7 \\
\hline & Traumatismo cerebral focal & 4 & 1,7 \\
\hline & Hemorragia subaracnoidea no especificada & 1 & 0,4 \\
\hline \multirow{3}{*}{ Severidad del trauma al ingreso (escala de Glasgow) } & Leve & 175 & 78,1 \\
\hline & Moderado & 37 & 16,5 \\
\hline & Grave & 12 & 5,3 \\
\hline \multirow{3}{*}{ Conducta } & Observación & 113 & 50,4 \\
\hline & Hospitalización & 57 & 25,4 \\
\hline & Cirugía & 54 & 24,1 \\
\hline \multirow{2}{*}{ Realizaron neuroimagen } & Sí & 125 & 55,8 \\
\hline & No & 99 & 44,1 \\
\hline \multirow{2}{*}{ Ingresó a UCE *- UCI † } & Sí & 47 & 20,9 \\
\hline & No & 177 & 79,0 \\
\hline Estancia hospitalaria en días (media-DE) & UCE-UCI & 43 & 19,1 \\
\hline \multirow{4}{*}{ Complicaciones } & Sensitiva & 0 & 0,0 \\
\hline & Motora & 5 & 2,2 \\
\hline & Sensitiva y motora & 4 & 1,7 \\
\hline & Ninguna & 215 & 95,9 \\
\hline \multirow{2}{*}{ Condición final } & Muerto & 1 & 0,4 \\
\hline & Vivo & 223 & 99,5 \\
\hline
\end{tabular}

* Unidad de cuidados especiales. † Unidad de cuidados intensivos. Fuente: creación propia 
En el análisis bivariado se encontró una diferencia significativa entre el TCE moderado/grave y el leve en cuanto a nivel socioeconómico bajo (valor $\mathrm{p}=0,000$ ), la atención en el Hospital General de Medellín (valor p =0,000) y no tener acompañante (valor $\mathrm{p}=0,002$ ). No se encontró diferencia con las variables sexo y edad (Tabla 3).

Tabla 3. Características de los niños con traumatismo craneoencefálico, según la severidad. Hospital General de Medellín y Clínica Somer de Rionegro, 2010-2017

\begin{tabular}{|c|c|c|c|c|c|c|c|}
\hline & & \multicolumn{5}{|c|}{ Escala Glasgow, n (\%) } & \multirow{3}{*}{ Valor-p } \\
\hline \multicolumn{2}{|c|}{ Característica } & \multirow[t]{2}{*}{$n=224$} & \multicolumn{2}{|c|}{ Leve } & \multicolumn{2}{|c|}{ Moderado/Grave } & \\
\hline & & & $\mathbf{n}$ & $\%$ & $\mathbf{n}$ & $\%$ & \\
\hline \multirow{2}{*}{ Sexo } & Femenino & 79 & 63 & 79,7 & 16 & 20,3 & \multirow{2}{*}{0,665} \\
\hline & Masculino & 145 & 112 & 77,2 & 33 & 22,8 & \\
\hline \multirow{3}{*}{ Edad } & $0-2$ años & 68 & 57 & 83,8 & 11 & 16,2 & \multirow{3}{*}{0,127} \\
\hline & 3-5 años & 65 & 53 & 81,5 & 12 & 18,5 & \\
\hline & 6-10 años & 91 & 65 & 71,4 & 26 & 28,6 & \\
\hline \multirow{2}{*}{ Estrato socioeconómico bajo } & No & 134 & 118 & 88,1 & 16 & 11,9 & \multirow{2}{*}{0,000} \\
\hline & Sí & 90 & 57 & 63,3 & 33 & 36,7 & \\
\hline \multirow{2}{*}{ Sitio de atención } & Clínica SOMER & 129 & 124 & 96,1 & 5 & 3,9 & \multirow{2}{*}{0,000} \\
\hline & Hospital General de Medellín & 95 & 51 & 53,7 & 44 & 46,3 & \\
\hline \multirow{2}{*}{ Tiene acompañante $(n=221)^{*}$} & Sin acompañante & 23 & 12 & 52,2 & 11 & 47,8 & \multirow{2}{*}{0,002} \\
\hline & Con acompañante & 198 & 160 & 80,8 & 38 & 19,2 & \\
\hline
\end{tabular}

*Datos de historias clínicas que tenían información sobre la presencia de acompañante. Fuente: creación propia

\section{DISCUSIÓN}

EI TCE en la población pediátrica es un problema de salud pública que afecta a todos los grupos demográficos, independientemente de la ubicación o el estrato socioeconómico ${ }^{(14,29)}$. Este estudio describe los principales aspectos sociodemográficos, circunstanciales, espacio-temporales y clínicos de los niños menores de 10 años de edad que fueron atendidos por TCE en dos instituciones de salud de referencia en Medellín y Rionegro, se obtuvieron resultados que enriquecen la epidemiología de este tipo de trauma en el país.

Existen diferentes mecanismos que pueden causar un TCE en pediatría. En la presente investigación se encontró como principal mecanismo de lesión las caídas, seguido por el accidente de tránsito y maltrato físico.

Según otros estudios las causas están determinadas por la edad, con mayor frecuencia en los grupos menores de 5 años y mayores de 15 años, siendo las caídas y los accidentes automovilísticos los más frecuentes, respectivamente ${ }^{(7,21,30)}$. En un metaanálisis del grupo IMPACT (del inglés International Mission on Prognosis and Analysis of Clinical Trials in TBI) describieron como causa principal el accidente automovilístico seguido por las caídas y los accidentes deportivos en pacientes mayores de 14 años ${ }^{(31)}$. Sin embargo, en otros lugares geográficos como África, Oriente medio y el sur de Asia se reportan las caídas como la principal causa del TCE, seguidas de accidentes de tránsito y trauma no accidental ${ }^{(5,32-34)}$. Según Michael et al., estas variaciones demográficas pueden estar dadas por el nivel de desarrollo del país ${ }^{(14)}$.

La población pediátrica tiene características sociodemográficas que los predisponen a sufrir un TCE, entre estas se encuentran los grupos etarios. En los lactantes y preescolares las condiciones anatómicas, como una mayor masa craneofacial y la falta de desarrollo psicomotor los hace más propensos a sufrir caídas ${ }^{(17,28,35)}$. En los escolares y adolescentes los roles sociales y tipos de actividades los hacen más susceptibles a traumas por 
caídas, accidentes de tránsito o violencia ${ }^{(21,36-39)}$. En el presente estudio se encontró una distribución similar para los tres grupos de edad, en Asia y Estados Unidos se ha descrito que los lactantes y adolescentes presentan una mayor frecuencia de TCE ${ }^{(5,14,30)}$. En el Hospital La Misericordia de Bogotá reportaron en 2015 que los Escolares fueron afectados en un 30,2 \%, los Lactantes en un 27,2 \% y los Preescolares en un 23,6\% ${ }^{(22)}$; la misma distribución por estos grupos etarios fue descrita en otras investigaciones ${ }^{(6,19,21)}$.

Los sitios de ocurrencia varían también según el grupo etario, en menores de 5 años los traumas ocurren más frecuentemente en el domicilio, en los Escolares y Adolescentes predominan las zonas de actividad física ${ }^{(21,40)}$; estos datos son similares a los de otras investigaciones $^{(14,41)}$.

Con respecto al sexo la población masculina es la más afectada con el $70 \%$ de los casos ${ }^{(8,30,37,42,43)}$, lo cual concuerda con los datos obtenidos en esta investigación. Esta diferencia podría explicarse por el tipo de actividades físicas y los comportamientos propios de cada $\operatorname{sexO}{ }^{(44,45)}$.

EI nivel socioeconómico representa un factor de riesgo para sufrir un TCE. En una investigación realizada en el Reino Unido se describió que los niveles socioeconómicos bajos experimentan tasas más altas de TCE ${ }^{(4)}$. En Ecuador se evidenció que el 51,8 \% de los pacientes que sufrieron un TCE eran de Nivel socioeconómico mediobajo y bajo ${ }^{(41)}$, para Arango et al., el 69 \% de la población estudiada provenía de Niveles socioeconómicos bajos ${ }^{(24)}$; en esta investigación se encontraron datos similares. Por otro lado para Thurman no existe suficiente información con respecto al estrato socioeconómico en los artículos existentes para definir esta predisposición ${ }^{(30)}$

La gravedad del TCE se puede evaluar mediante la escala de coma de Glasgow al momento de la consulta y durante la observación clínica ${ }^{(10)}$. Este estudio mostró una mayor frecuencia de TCE leve, seguido de TCE moderado y grave, esta misma distribución se ha encontrado en la literatura. En una revisión sistemática desde 1990 hasta 2015 de la Universidad de Nashville, el TCE leve representó el 80 \% de los casos y el TCE grave del 3 al $7 \%{ }^{(30)}$, en Colombia el TCE leve se ha encontrado en un 68,6 \% de los casos, moderado $25 \%$ y grave 5,3\%, con una mortalidad inferior al $0,6 \%{ }^{(21-26,44)}$. Las contusiones son las manifestaciones más frecuentes generadas por un TCE leve y hasta en un $90 \%$ no se encuentran hallazgos patológicos en las neuroimágenes ${ }^{(14,46)}$; en un TCE moderado o grave se pueden encontrar lesiones como las fracturas craneanas, contusiones cerebrales o hematomas intracerebrales que son identificadas con tomografías craneales ${ }^{(5,47-49)}$.

En esta investigación las contusiones fueron las lesiones más frecuentes, seguidas por las fracturas craneanas y hemorragia epidural, datos similares describen Dewan et al., quienes encontraron que las contusiones representaron del 15 al $61 \%$ y, la fractura de cráneo del 19 al $45 \%$, los hematomas subdurales un $12 \%$ y los epidurales $19 \%{ }^{(14)}$. Según la condición clínica del niño se determina el sitio de atención intrahospitalario, el $60 \%$ de los pacientes requieren observación en el servicio de urgencias, ingresan a hospitalización el 30 \% y necesitan atención en la unidad de cuidado intensivo (UCI) entre el $10-20 \%{ }^{(11,44)}$. En este estudio la necesidad de ingreso a la UCI se presentó con mayor frecuencia a lo reportado, lo cual podría corresponder a que uno de los hospitales del estudio cuenta con UCI pediatría $y$ es centro de referencia en la ciudad.

EI daño cerebral producto del TCE en la infancia puede ser variable, incluyendo alteraciones físicas, cognitivas, comportamentales y sociales que van a interferir en las actividades cotidianas, educativas e intrafamiliares de los afectados ${ }^{(15,50-52)}$. Esta alteración depende de factores como el tipo de lesión y la severidad del traumatismo ${ }^{(52-54)}$.

Di Batista et al., evidenciaron que la gravedad deI TCE influye significativamente en la calidad de vida del paciente, siendo las lesiones leves las que se asocian con un buen pronóstico, pero las lesiones moderadas y severas se han asociado a una baja calidad de vida ${ }^{(55,56)}$. Entre los trastornos emocionales y conductuales asociados al TCE está la depresión, la ansiedad e irritabilidad ${ }^{(54,57,58)}$; datos similares reporta la Universidad de Melbourne en Australia ${ }^{(55)}$.

Con respecto al área cerebral comprometida se presentan diferentes manifestaciones, en el caso del lóbulo frontal o temporal puede ocasionar dificultad para regular o controlar la conducta, déficit en la memoria y alteraciones sociales y físicas ${ }^{(13,15,52,59,60)}$.

En el 2017 se realizó una revisión sistemática de la calidad de vida relacionada con la salud en los niños 
latinoamericanos con TCE, se encontró que los pacientes que sufrieron TCE moderado-severo se ven afectados en su calidad de vida durante los años siguientes al daño cerebral, siendo la esfera psicosocial la más afectada, esta se manifiesta en problemas escolares tanto de relaciones interpersonales como de rendimiento académico ${ }^{(61)}$.

En Bogotá un estudio reportó que la mayor parte de la población estudiada presentó algún grado de alteración funcional, sin que esto afecte significativamente sus actividades cotidianas, en los casos de niños que sufrieron TCE moderado o grave ${ }^{(19)}$; en este estudio se encontraron secuelas sensitivas y motoras que podrían comprometer la funcionalidad de los pacientes, pero no fue posible aplicar escalas durante la estancia hospitalaria ni un seguimiento a largo plazo, sin embargo puede ser una oportunidad para futuras investigaciones.

Una de las limitaciones de este estudio fue la inclusión de dos instituciones con características y población diferente, lo que dificulta profundizar en algunos análisis; el uso de fuentes secundarias de las cuales algunas carecían de información relevante en la investigación, adicionalmente, el grado de severidad o escala de Glasgow no pudo ser recolectado de manera cuantitativa y no hubo posibilidad de hacer un seguimiento posterior al paciente para evaluar secuelas.

Para terminar, es claro que el TCE es una preocupación de salud pública importante a nivel mundial. En diferentes partes del mundo se han desarrollado investigaciones sobre el TCE en la población pediátrica que han permitido tener una aproximación a la epidemiología de ciertas características propias de esta edad. Sin embargo, es notoria la falta de estudios que permitan caracterizar detalladamente esta población. En Colombia se encuentran reportes de diferentes ciudades al respecto, no obstante no se alcanza a dar cuenta de la epidemiología nacional.

Para la ciudad de Medellín falta ampliar los grupos de edad y tamaños de muestras que permitan determinar las condiciones, tanto clínicas como sociodemográficas de los pacientes pediátricos que sufren TCE. Adicionalmente hay una carencia de estudios que evalúen el curso de la enfermedad y las secuelas generadas por eI TCE, lo anterior no permite desarrollar un plan de rehabilitación física intra y extrahospitalaria y de acompañamiento psicosocial.

\section{CONFLICTOS DE INTERESES}

Ninguno por declarar.

\section{REFERENCIAS BIBLIOGRÁFICAS}

1. Menon DK, Schwab K, Wright DW, Maas AI. Position Statement: Definition of Traumatic Brain Injury. Arch Phys Med Rehabil. 2010;91(11):1637-40. DOI 10.1016/j. apmr.2010.05.017.

2. Pervez M, Kitagawa RS, Chang TR. Definition of Traumatic Brain Injury, Neurosurgery, Trauma Orthopedics, Neuroimaging, Psychology, and Psychiatry in Mild Traumatic Brain Injury. Neuroimag Clin N Am. 2018;28(1):1-13. DOI 10.1016/j.nic.2017.09.010.

3. Ha G, Jeon MJ, Sakong J. Analysis of causes of injuries among children in Daegu, Korea. Korean J Pediatr. 2010;53(11):942-50. DOI 10.3345/kjp.2010.53.11.942.

4. Parslow RC, Morris KP, Tasker RC, Forsyth RJ, Hawley CA. Epidemiology of traumatic brain injury in children receiving intensive care in the UK. Arch Dis Child. 2005;90(11):1182-7. DOI 10.1136/adc.2005.072405.

5. Shao J, Zhu H, Yao H, Stallones L, Yeates K, Wheeler K, et al. Characteristics and Trends of Pediatric Traumatic Brain Injuries Treated at a Large Pediatric Medical Center in China, 2002-2011. PLoS One. 2012;7(12):e51634. DOI 10.1371/journal.pone.0051634.

6. Paulsen Sandi DK, Mejia Salas H. Factores de riesgo para accidentes en niños Hospital deI Niño "Dr. Ovidio Aliaga Uria”. Arch Pediatr Urug. 2005;76(1):65-74.

7. Wu X, Hu J, Zhuo L, Fu C, Hui G, Wang Y, et al. Epidemiology of traumatic brain injury in eastern China, 2004: A prospective Iarge case study. J Trauma. 2008;64(5):1313-9. DOI 10.1097/TA.0b013e318165c803.

8. Chaitanya K, Addanki A, Karambelkar R, Ranjan R. Traumatic brain injury in Indian children. Childs Nerv Syst. 2018;34(6):1119-23. DOI 10.1007/s00381018-3784-z.

9. Fiorentino J, Molise C, Stach P, Cendredo P, Maía S, Hoffman E, et al. Trauma en pediatría. Estudio epidemiológico en pacientes internados en el Hospital de Niños " Ricardo Gutiérrez". Arch Argent Pediatr. 2015;113(1):1220. DOI 10.1590/S0325-00752015000100004.

10. Singh B, Murad MH, Prokop LJ, Erwin PJ, Wang Z, Mommer SK, et al. Meta-analysis of glasgow coma 
Scale and simplified motor score in predicting traumatic brain injury outcomes. Brain Inj. 2013;27(3):293300. DOI 10.3109/02699052.2012.743182.

11. Zaninotto ALC, Costa BT, Ferreira IS, French M, Paiva WS, Freoni F. Traumatic brain injury. En: Freoni F, editor. Clinical Trials in Neurology. Neuromethods. New Zork: Humana Press Inc; 2018. p. 105-38.

12. Appenteng R, Nelp T, Abdelogadir J, Weledji N, Haglund M, Smith E. A systematic review and quality analysis of pediatric traumatic brain injury clinical practice guidelines. PLoS One. 2018;13(8):e0201550. DOI 10.1371/journal.pone.0201550.

13. Vázquez-Solís MG, Villa-Manzano AI, Sánchez-Mosco DI, Vargas-Lares JJ, Plascencia-Fernandez I. Pronóstico del traumatismo craneoencefálico pediátrico Estudio de una cohorte dinámica. Rev Med Inst Mex Seguro Soc. 2013;51(4):372-7.

14. Dewan MC, Mummareddy N, Wellons JC, Bonfield CM. Epidemiology of global pediatric traumatic brain injury: a qualitative review. World Neurosurg. 2016;91(1):497-509. DOI 10.1016/j.wneu.2016.03.045.

15. Barlow KM, Thomson E, Johnson D, Minns RA. Late Neurologic and Cognitive Sequelae of Inflicted Traumatic Brain Injury in Infancy. Pediatrics. 2005;116(2):e174. DOI 10.1542/peds.2004-2739.

16. Keyes KM, Susser E, Pilowsky DJ, Hamilton A, Bitfoi A, Goelitz D, et al. The health consequences of child mental health problems and parenting styles: Unintentional injuries among European schoolchildren. Prev Med. 2014;67(7):182-8. DOI 10.1016/j.ypmed.2014.07.030.

17. Chaudhary S, Figueroa J, Shaikh S, Mays EW, Bayakly $R$, Javed $M$, et al. Pediatric falls ages $0-4$ : understanding demographics, mechanisms, and injury severities. Inj Epidemiol. 2018;5(SuppI 1):7. DOI 10.1186/ s40621-018-0147-X.

18. Spitz G, Mckenzie D, Attwood D, Ponsford JL. Cost prediction following traumatic brain injury : model development and validation. J Neurol Neurosuro Psychiatry. 2016;87(2):173-80. DOI 10.1136/jnnp-2014-309479.

19. MRC CRASH Trial Collaborators, Perel P, Arango M, Clayton T, Edwards P, Komolafe E, et al. Predicting outcome after traumatic brain injury: practical prognostic models based on large cohort of international patients. BMJ. 2008;336(7641):425-9. DOI 10.1136/ bmj.39461.643438.25.
20. Centers for Disease Control and Prevention [internet]. Rates of TBI-related Emergency Department Visits by Age Group-United States, 2001-2010. EEUU: CDC; 2016. [Consultado 2018 nor 3]. Disponible en: http://cort.as/-M940

21. Catellanos NJ. Caracterización sociodemográfica y clínica de los casos de trauma craneoencefálico infantil atendidos en una institución pediátrica entre 2012 y 2014. [Trabajo de grado Especialista en Neurocirugía]. Bogotá: Universidad Nacional de Colombia; 2015.

22. Wilches Bornacelly L, Barbosa Álvarez AC, Flórez J, Cogollos Amaya A, Flórez Medina G. Caracterización del trauma pediátrico en un hospital militar de la ciudad de Bogotá. Revista Med. 2015;23(2):50-9.

23. Uribe Castrillón LM, Fernandez Laverde M. Manejo del trauma encefalocraneano en niños en servicios de Urgencias pediátricas. Unidades de Uroencias de Medellín. Enero - junio de 2008. Med UPB. 2009;28(1):17-21.

24. Arango Soto D, Quevedo Vélez A, Montes Gallo A, Cornejo Ochoa JW. Epidemiología del trauma encefalocraneano (TEC) en 90 lactantes mayores $y$ preescolares atendidos en un servicio de urgencias pediátricas de tercer nivel, en Medellín, Colombia. IATREIA. 2008;21(3):271-9.

25. Betancur NL, López DC, Patiño AM, Muñoz A, Castaño JR, Rodríouez DF, et al. Universidad de Manizales, principales causas del trauma craneoencefálico en niños. Hospital infantil universitario. Enero a julio del 2002 [internet]. [Consultado 8 oct 2018]. Disponible en: http://cort.as/-N_WC.

26. Correa MA, González G, Herrera MH, Orozco A. Epidemiología del trauma pediátrico en Medellín, CoIombia 1992-1996. Colomb Med. 2000;31(2):77-80.

27. Oroganización Panamericana de la Salud. Clasificación estadística internacional de enfermedades y problemas relacionados con la salud: 10a revisión [internet]. [Consultado 8 oct 2018]. Disponible en: http://cort.as/-MEqq

28. Posada A, Gomez JF, Ramirez H. Crecimiento y desarrollo. En: Posada A, Gomez JF, Ramirez H, editors. EI niño sano. 4a ed. Medellin: Panamericana; 2016. p. 114-53.

29. Faul M, Xu L, Wald M, Coronado V. Traumatic Brain Injury in the United States: emergency department 
visits, hospitalizations, and deaths 2002-2006. U.S. Atlanta: National Center for Injury Prevention and Control; 2010.

30. Thurman DJ. The Epidemiology of Traumatic Brain Injury in Children and Youths: A Review of Research Since 1990. J Child Neurol. 2016;31(1):20-7. DOI $10.1177 / 0883073814544363$.

31. Butcher I, Mchugh G, Lu J, Marmarou A, Maas AI, Mushkudiani N, et al. Prognostic Value of Cause of Injury in Traumatic Brain Injury: Results from The IMPACT Study. J Neurotrauma. 2007;24(2):281-6. DOI 10.1089/neu.2006.0030.

32. Işik HS, Gökyar A, Yildiz Ö, Bostanci U, Özdemİ C. Pediatric head injuries, retrospective analysis of 851 patients: an epidemiological study. Ulus Travma Acil Cerrahi Dero. 2011;17(2):166-72.

33. Zhu H, Gao Q, Xia X, Xiang J, Yao H, Shao J. ClinicallyImportant Brain Injury and CT Findings in Pediatric Mild Traumatic Brain Injuries : A Prospective Study in a Chinese Reference Hospital. Int J Environ Res Public Health. 2014;11(4):3493-506. DOI 10.3390/ijerph110403493.

34. Chan H, Aasim W, Abdullah N, Naing N, Abdullah J, Saffari $M$, et al. Characteristics and clinical predictors of minor head injury in children presenting to two Malaysian accident. Singapore med J. 2005;46(5):219-23.

35. Sims A, Chounthirath T, Jingzhen $Y$, Nichole L, Smith GA. Infant Walker - Related Injuries in the United States. Pediatrics. 2018;142(4):e20174332. DOI 10.1542/ peds.2017-4332.

36. Silveira Franciozi CE, Sugawara Tamaoki MJ, Alves de Araújo EF, Dobashi E, Utumi CE, Pinto JA et aI. Epidemiology, Treatment and Economical Aspects Multiple of Trauma in Children and Adolescents in a Public Hospital. Acta Ortop Bras. 2008;16(5):261-5. DOI 10.1590/S1413-78522008000500001.

37. Durkin M, Olsen MB. The epidemiology of urban pediatric neurological trauma: Evaluation of, and implications for, injury prevention programs. Neurosurgery. 1998;42(2):300-10. DOI 10.1097/00006123199802000-00052.

38. Alcoholado BI. Trauma pediátrico, ihay diferencias? Rev chil pediatr. 2000;71(4):357-60. DOI 10.4067/ S0370-41062000000400014.

39. Montoya B, Espinosa T, Arias R. Lesiones accidentales. En: Radiografía de la violencia regional. Énfasis en niños, niñas y adolescentes: indicadores de diversos tópicos de violencia en el departamento de Antioquia 2015. $2^{\text {a }}$ ed. Medellín: Corporación Universitaria Remington; 2017. p. 201-16.

40. Peeters W, van den Brande R, Polinder S, Brazinova A, Steyerberg EW, Lingsma HF, et al. Epidemiology of traumatic brain injury in Europe. Acta Neurochir (Wien). 2015;157(10):1683-96. DOI 10.1007/s00701-015-2512-7.

41. Pérez Zúñioga MF. Prevalencia de trauma encéfalocraneal en niños de 0 a 5 años y factores asociados, en el servicio de emeronencia pediátrica del HVCM febrero-julio de 2014. [Trabajo de grado Especialista en Pediatría]. Ecuador: Universidad de Cuenca, 2016.

42. Hillier S, Hiller J, Metzer J. Epidemiology of traumatic brain injury in South Australia. Brain Inj. 1997;11(9):649-59. DOI 10.1080/026990597123205.

43. Queiroz VA, Assis AM, Pinheiro SM, Ribeiro HC Jr. Predictors of linear growth in the first year of life of a prospective cohort of full term children with normal birth weight. J Pediatr (Rio J). 2012;88(1):79-86. DOI 10.2223.JPED.2143.

44. Bobenrieth F. Trauma de cráneo en pediatría conceptos, guias, controversias y futuro. Rev Med Clin Condes. 2011;22(5):545-695. DOI 10.1016/S07168640(11)70476-X.

45. Restrepo JM. Epidemiología y prevención del trauma en accidentes de tránsito. En: Quintero L, editor. Trauma. Abordaje inicial en los servicios de uroencias. Cali: Salamandra; 2008; p. 51-62.

46. Mallicote MU, Isani MA, Golden J, Ford HR, Upperman JS, Gayer CP. Screening for blunt cerebrovascular injuries in pediatric trauma patients. J Pediatr Suro. 2019;S0022-3468(19)30311-2. DOI 10.1016/j. jpedsurg.2019.04.014.

47. Kim HB, Kim DK, Kwak YH, Shin SD, Song KJ, Lee $\mathrm{SC}$, et al. Epidemiology of Traumatic Head Injury in Korean Children. J Korean Med Sci. 2012;27(4):437-42. DOI 10.3346/jkms.2012.27.4.437.

48. Amaranath JE, Ramanan M, Reagh J, Saekang E, Prasad N, Chaseling R, et al. Epidemiology of traumatic head injury from a major paediatric trauma centre in New South Wales, Australia. ANZ J Suro. 2014;84(6):424-8. DOI 10.1111/ans.12445.

49. Crowe L, Babl F, Anderson V, Catroppa C. The epidemiology of paediatric head injuries: Data from a referral centre in Victoria, Australia. J Paediatr 
Child Health. 2009;45(6):346-50. DOI 10.1111/j.14401754.2009.01499.x.

50. Ryan NP, Catroppa C, Godfrey C, Noble-Haeusslein LJ, Shultz SR, O'Brien TJ, et al. Social dysfunction after pediatric traumatic brain injury: A translational perspective. Neurosci Biobehav Rev. 2016;64:196-214. DOI 10.1016/j.neubiorev.2016.02.020.

51. Wells R, Minnes P, Phillips M. Predicting social and functional outcomes for individuals sustaining paediatric traumatic brain injury. Dev Neurorehabil. 2009;12(1):12-23. DOI 10.1080/17518420902773109.

52. Shultz EL, Hoskinson KR, Keim MC, Dennis M, Taylor HG, Bigler ED, et al. Adaptive Functioning Following Pediatric Traumatic Brain Injury : Relationship to Executive Function and Processing Speed. Neuropsychology. 2016;30(7):830-40. DOI 10.1037/neu0000288.

53. Ciccia AH, Beekman L, Ditmars E. A clinically focused systematic review of social communication in pediatric TBI. NeuroRehabilitation. 2018;42(3):331-344. DOI 10.3233/NRE-172384.

54. Arciniegas DB, Wortzel HS. Emotional and behavioral dyscontrol after traumatic brain injury. Psychiatr Clin N Am. 2014;37(1):31-53. DOI 10.1016/j. psc.2013.12.001.

55. Di Battista A, Soo C, Catroppa C, Anderson V. Quality of Life in Children and Adolescents Post-TBI: A Systematic Review and Meta-Analysis. J Neurotrauma. 2012;29(9):1717-27. DOI 10.1089/neu.2011.2157.
56. Rodríguez Moya VS, Tena Cabalé M, Viamontes Martínez WA, Gallo Borrero CM, Montejo Montejo J, Pérez Morales Y. Evaluacion de las secuelas intelectuales en niños con trauma craneoencefalico severo. Rev Cubana Pediatr. 2014;86(3):336-43.

57. Duhaime A, Beckwith J, Maerlender A, Mcallister T, Crisco J, et al. Spectrum of acute clinical characteristics of diagnosed concussions in college athletes wearing instrumented helmets. J Neurosurg. 2012;117(6):1092-9. DOI 10.3171/2012.8.JNS1 12298.

58. Baker JM, Good DE. Physiological emotional underarousal in individuals with mild head injury. Brain Inj. 2014;28(1):51-65. DOI 10.3109/02699052.2013.857787.

59. De Freitas Cardoso MG, Faleiro RM, de Paula JJ, Kummer A, Caramelli P, Teixeira AL, et. al. Cognitive Impairment Following Acute Mild Traumatic Brain Injury. Front. Neurol. 2019;10:198. DOI 10.3389/ fneur.2019.00198.

60. Ichkova A, Rodriguez-Grande B, Bar C, Villega F, Konsman JP, Badaut J. Vascular impairment as a pathological mechanism underlying long-lasting cognitive dysfunction after pediatric traumatic brain injury. Neurochem Int. 2017;111:93-102 DOI 10.1016/j. neuint.2017.03.022.

61. Gutierrez K, Luna A, Mosquera V. Revisión sistemática de la calidad de vida relacionada con la salud en niños latinoamericanos con trauma craneoencefálico. Acta Neurol Colomb. 2017;33(4):286-98. DOI 10.22379/24224022169. 